\title{
Neurobiology of Posttraumatic Stress Disorder (PTSD) and its Frontostriatal Implications: a short review
}

\author{
Hugo Sánchez-Castillo ${ }^{1}$ \\ Martin Migliaro ${ }^{5}$ \\ National Autonomous University of Mexico, Mexico
}

\author{
Diana Berenice Paz-Trejo ${ }^{2}$ \\ Josselyn Vazquéz Ramirez ${ }^{3}$ \\ Pavel Zarate González ${ }^{4}$ \\ Ibero-American Society of Applied Neuroscience
}

\begin{abstract}
Throughout its evolutionary course, stress has remained as an adaptive response to stimuli that may jeopardize the integrity of an organism. Within this perspective, we can classify the stressors as psychological, physical or harmful to cardiovascular stability. However, when intense stressful events occur, there is a possibility of developing PTSD. This disorder makes use of the hypothalamic-pituitary-adrenal axis, which is commonly activated during stress and is kept activated even when the stressful stimulus has ended months ago. The consequences of this condition are observed at the neuroendocrine, neurochemical and anatomical level. This review aims to give a brief report of the neurobiology of stress, PTSD, and its implications in various structures, such as the amygdala, hippocampus and prefrontal cortex.
\end{abstract}

Keywords. Stress, Cortisol, Trauma, hippocampus, prefrontal cortex.

\footnotetext{
${ }^{1}$ Hugo Sanchez-Castillo, National Autonomous University of Mexico and Ibero-American Society of Applied Neuroscience. Postal address: Laboratorio de Neuropsicofarmacologia y Estimación Temporal, Facultad de Psicología, Universidad Nacional Autónoma de México, Av Universidad 3000, Col Copilco Universidad, México. E-mail: ajuscoman@gmail.com

${ }^{2}$ Diana Berenice Paz-Trejo, Ibero-American Society of Applied Neuroscience. E-mail: dianabpt@gmail.com

${ }^{3}$ Josselyn Vazquéz Ramirez, Ibero-American Society of Applied Neuroscience. E-mail: joselyn.psicologia@gmail.com

${ }^{4}$ Pavel Zarate Gonzalez, Ibero-American Society of Applied Neuroscience. E-mail: pavelzar@gmail.com

${ }^{5}$ Martin Migliaro, National Autonomous University of Mexico. E-mail: martinmigliaro@yahoo.com
} 


\section{Introduction}

\section{Stress and Hypothalamic-Pituitary-Adrenal Axis}

Currently stress is one of the most common ailments of our society. Stress naturally allows us to survive and properly adapt in situations that may jeopardize the survival of an individual, its offspring, territory or the entire species. However, it has been reported that intense stressful events (natural disasters, military combat, sexual abuse or domestic violence) can lead to Post Traumatic Stress Disorder (PTSD), which affects (according to international figures) about $8-14 \%$ of the global population (Bremner, 2006; Bailey, Cordell, Sobin, \& Neumeister, 2013). Furthermore, this condition has been found to have a comorbid association with depression (Womble, 2013), anxiety (Keltner \& Dowben, 2007), substance abuse (Mills, 2013), dissociative disorder (Lanius et al, 2010) etc. Currently, PTSD has been described as the result of a chronic anxiety disorder (Bailey et al., 2013).

It has been reported that some of the physiological changes associated with stress include: 1) disposition of energy in order to maintain the functions of muscles and the central nervous system (CNS);2) increased attentional resources directed to the stressor stimulus; 3 ) increased heart and respiratory rate; 4) redistribution of blood flow toward muscles and the CNS; 5) modulation of the immune system; 6) down regulation of the reproductive sexual response; 7) loss of bowel control; 8) decreased food intake and appetite (Carrasco \& Van de Kar, 2003). These coordinated responses disrupt the operations of the system so that the individual's probability of survival is increased. Within this perspective the stressor can be defined as the condition of danger or perceived danger that threatens the survival of the individual. Generally these stressors can be grouped into three categories: 1) psychological stressors that may be based on learned responses to the threat of an adverse condition (fear, anxiety, etc.); 2) stressors involving a physical stimulus with a strong psychological component (pain, electric shock, immobilization, etc.) and 3) stressors that threat cardiovascular homeostasis (hemorrhage, bleeding, exercise, heat exposure, etc.) (Van de Kar \& Blair, 1999).
Primarily, stress and PTSD have been associated with overactivation of the hypothalamic-pituitary-adrenal axis (HPA). The HPA axis has been reported to activate the neuroendocrine system in order to maintain an adaptive state to stress and the maintenance of physiological regulatory systems after the stressful event, thus ensuring survival (Bremner, 2005). Furthermore it has also been shown that a dysfunctional state of the HPA axis has been associated with various psychiatric and psychomotor disorders (Womble, 2013; Humphreys, Sauder, Martin \& Marx, 2009). At the hypothalamic level, the corticotrophin releasing factor (CRF) is liberated in response to stress, resulting in an activation of the HPA and the consequent increase in cortisol. CRF acts by means of the $G$ protein-coupled receptors, CRF-1 and CRF-2. It has been reported that high levels of CRF during the moment of a traumatic experience facilitates the encoding of a traumatic memory and maintains durable the effects of anxiety due to the stimulation of the CRF - 1 receptor (Hubbard, Nakashima, Lee \& Takahashi, 2007). Figure 1 shows a simplified diagram of the basic stress response without involving additional cortical mechanisms (see Figure 1).

\section{Amygdala}

Classically the amygdala is one of the structures related to the processing of emotions and it is evidently greatly related to stress. The amygdala is composed of multiple nuclei that execute different functions. The basolateral and lateral nuclei allow sensory integration coming from the thalamus, as well as cognitive information from the cerebral cortex and hippocampus (Van de Kar \& Blair, 1999). On the other hand, the central nucleus of the amygdala is related to behavioral, endocrine, and autonomous responses (Van de Kar \& Blair, 1999). Furthermore, the amygdala is also innervated by the raphe nuclei and the catecholaminergic brainstem nuclei, which in turn activate the CRF receptors in the paraventricular nucleus of the hypothalamus (Petrov et al., 1994).

Several studies have reported an increase in amygdaloid function under conditions of PTSD (Shin et al,. 2004); sounds and smells related to combat (Vermetten, Schmahl, Southwick \& Bremner, 2007), 


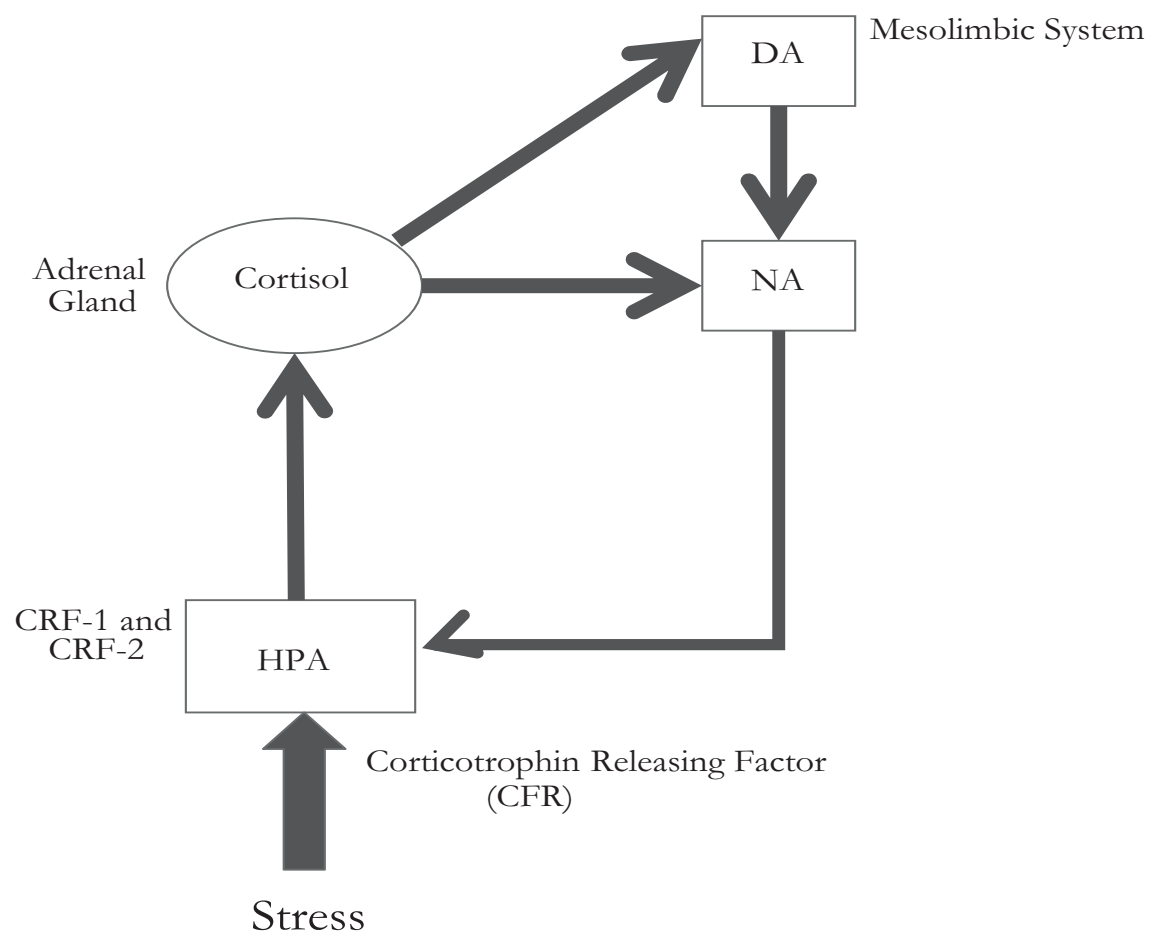

Figure 1. Basic outline of stress activation at hypothalamic-pituitary-adrenal (HPA) level. It can be seen that the active axis causes changes in stress and dopamine(DA) and noradrenaline (NA) systems.

photographs of events related to the traumatic events or words (Protopopescu et al., 2005), fear conditioning (Bremner et al., 2005), and facial expressions of fear (Shin et al., 2005). Even this overactivation of the amygdala has been found in PTSD patients during rest and during the execution of neutral attention and memory tasks (Shin et al., 2004).

\section{Hippocampus}

Anatomically, hippocampus is located adjacent to the amygdala and is involved in processes of working memory, spatial memory, and the categorization of stimuli. The proper functioning of the hippocampus is critical for explicit or declarative memory (Squire \& Zola-Morgan, 1991). This structure is related to the evaluation of stimuli and how these are spatially and temporally related using previously stored information.
Henceforth, this allows the system to determine in which ways stimuli are associated: that is, if they involve punishment, reward, novelty, or no-reward. The decrease of hippocampal function produces behavioral desensitization and hyperresponsiveness to environmental stimuli (Shestatzky, Greenberg \& Lerer, 1988). This hippocampal function has the additional component of involving the serotonergic system, as it has shown that this system plays a role in activation or inhibition of pathways that prevent the onset of stress responses. This has been complemented by the findings that animals with elevated corticosterone induced by stress manifested a decrease of hippocampal function (Pfaff, Silva \& Weiss, 1971). High levels of glucocorticoids have negative effects on memory resulting in a subsequent death of hippocampal cells if maintained for prolonged periods (Uno et al., 1994). 


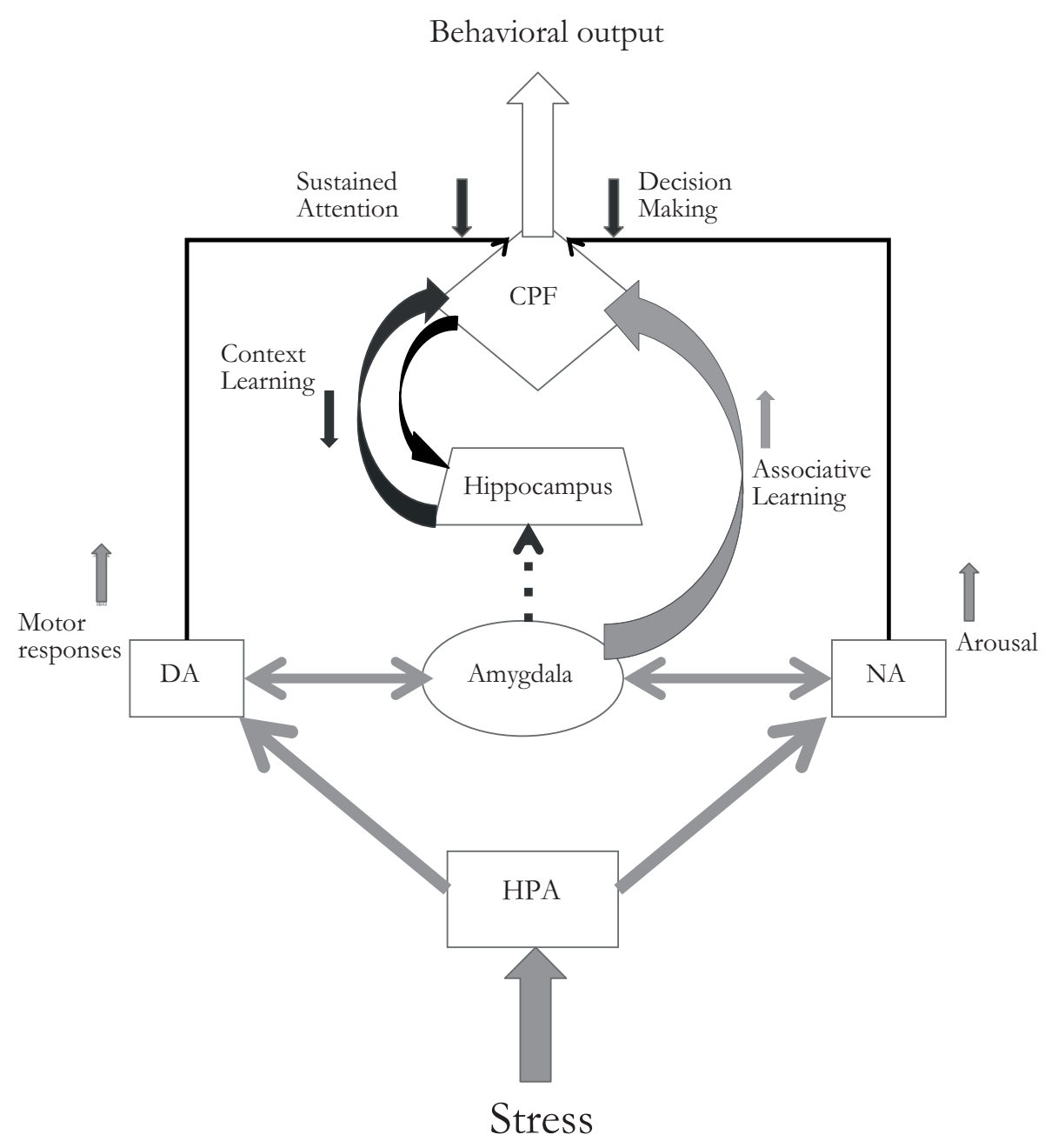

Figure 2. Outline of the effects on the prefrontal cortex.

It has been reported that people with PTSD show a drop in hippocampal volume (Zhang et al., 2011). In one study, Bremmer (1995) showed that Vietnam veterans with PTSD had a hippocampal reduction of $8 \%$ in the right hemisphere compared to veterans who did not show PTSD. In women with a history of being abused during childhood and having PTSD, it was observed a reduction of 5\% (Stein, Koverola, Hanna, Torchia \& McClarty, 1997). The most dramatic evidence comes from the study by Gurvits et al., (1996), whom reported that Vietnam veterans with severe combat exposure and PTSD had a severe hippocampal shrinkage percentage of $26 \%$ in the left hippocampus and $22 \%$ in the right hippocampus compared with veterans that did engage in combat but did not have symptoms of PTSD (Gurvits et al., 1996).

The shrinking of the hippocampus is related to loss of cells and could be due to dendritic atrophy or cell death, even though the mechanism is yet unclear. It has been shown that patients with PTSD perform poorly in verbal memory test. It has been found that it can be associated with the difficulty of distinguishing safe environments in PTSD patients. 


\section{Prefrontal Cortex and Neuromodulation}

Compared to acute stress, chronic stress produces more extensive alterations in the PFC of rats, including structural changes. Layers II and III lose dendritic materials: i.e. the length of dendrites and spine density are compromised (Holmes \& Wellman, 2009; Radley et al., 2006). The loss of spines is more evident in distal sites (200 $\mu \mathrm{m}$ from the soma approximately) and fungiform spines (probably related to the establishment of new connections) (Michelsen et al., 2007). These dendritic changes in the PFC of rats are associated with attentional dysfunction and deficits in working memory. In addition, chronic stress alters the plastic relationship between the PFC and the hippocampus (which is necessary for memory consolidation) (Arnsten, 2009). Behaviorally, rats show deficits in acquisition on the conditioned taste aversion task, as well in the radial maze task. However, there is still no conclusive evidence of the effects of chronic stress and its long-term implications.

Although stress has been primarily linked to the release of cortisol and stimulation of its receptors, there are neuromodulators that have been linked to stress and PTSD. Such is the case of noradrenaline (NA) and dopamine (DA). Noradrenergic and dopaminergic neurons in the brainstem change their firing rates according to the relevance of the events in the environment. Particularly noradrenergic cells during No-stress conditions have a pronounced phasic firing rate toward relevant stimuli, whereas the exposure to stressful stimuli presents a high tonic firing rate with phasic deregulation. This implies that the neurons fire to relevant stimuli while alert, but also to irrelevant stimuli during fatigue or stress (Aston-Jones, Rajkowski \& Cohen, 1999).

It has been described that the function that correlates the level of NA and working memory is represented by an inverted "U". This is possible because NA locks to different types of receptors depending on the amount released. The NA shows high affinity for alpha2 adrenoceptors and low affinity for alpha1 and beta adrenergic (Arnsten, 2000). It has been shown that the levels of NA that are released during alertness in non-stressing conditions is related to the activation of the alpha2 (Li \& Mei, 1994) while high levels of NA released during stress, which prevent the function of $\mathrm{CPF}$, are related to the stimulation of receptors of low affinity alpha1 beta1 (Birnbaum, Gobeske, Auerbach, Taylor \& Arnsten, 1999; Ramos et al., 2005). This implies that both the depletion of NA as blocking the receptor alpha 2 produces deficits in working memory, while the stimulation of the postsynaptic receptors of alpha2 in the PFC improves it. At the cellular level, it was found that blocking the alpha2 receptor reduces the firing rate in the PFC while its stimulation increases the firing rate (Li, Mao, Wang, \& Mei, 1999, Wang et al., 2007). By contrast, a high level of stimulation of alfa1 receptor in the PFC suppresses the firing rate and affects working memory. It has been shown that these deficits can be reversed by the administration of alpha1 antagonists administered systemically or directly into the PFC. These studies have been validated in humans since the administration alpha1 antagonist, prazosin, improves some cognitive deficits in PTSD (Peskind, Bonner, Hoff, \& Raskind, 2003; Raskind et al., 2002).

In the case of the dopaminergic cells, it has been recently described that they increase their firing rate when aversive stimuli are present and these changes may affect the functioning of the PFC during episodes of stress. These two systems (NA and DA) increase in the PFC during episodes of acute and medium stress. The PFC has been reported to have direct and indirect connections to cell bodies in the brain stem; as well in the locus coereleus (LC), substantia nigra $(\mathrm{SN})$ and ventral tegmental area (AVT); in addition these systems can regulate their own catecholaminergic entries. The optimal levels of catecholamine release allow the regulation of PFC operations, thus creating a functional cycle.

Under these highly stressful conditions, amygdala activates the "stress pathway" in the hypothalamus and brain stem, which produces high levels of NA and DA. This release affects the regulation of PFC, but increases the amygdaloid function, thus bringing a dysfunctional cycle. For example, high levels of catecholamines during stress produces fear conditioning mediated by 
the amygdala, while on the other hand, stress hinders cognitive abilities related with the PFC like working memory and attentional regulation. This is due to the fact that attentional regulation has a "top down" control by the PFC, which is based on what is most immediately relevant, and a "bottom up" control from the sensory cortex that assesses the salience of the stimuli that capture attentional resources. At the same time, the amygdala skews the reaction towards a quick emotional motor response during stressful episodes, rather than a slow response mediated by the PFC (Arnsten, 2009).

This shows us that the correct operation of the PFC gives us flexibility in the regulation of behavior to enable us to adapt to a changing environment, that is, allows us to "move" attentional resources to new dimensions in order to alter decision making according to the contingencies of the environment. The PFC monitors errors allowing introspection about what is inappropriate and changes strategies. All these skills depend on the functioning of PFC and its network of neural connections, which are highly sensitive to neurochemical environment.

\section{Conclusion}

The reduction of CPF operations occurs during high levels of stress and it is highly relevant to understanding the mental health of patients with such disorders. The loss of self-control during exposure to stress can bring along a large number of maladaptive behaviors, such as the consumption of stimulants (cocaine, amphetamines, etc.); excess consumption of tobacco, alcohol, overeating etc. Prolonged stress may be a risk factor for developing major depression and traumatic stress can lead to PTSD. Furthermore, stress can exacerbate the symptoms of schizophrenia and bipolar disorder. Given these effects on human health, it is vital to implement animal models under conditions of chronic stress and traumatic stress as a means to understand the mechanisms that keep them vulnerable (Arnsten, 2009).

Despite of the large deterioration caused by PTSD, our understanding of the pathophysiology that directs different pathways associated with severe stress remains uncertain, even current models have failed to provide more effective therapeutic targets. Considering that the presently recommended treatment for PTSD consists of psychological therapy and the administration of selective serotonin reuptake inhibitors (SSRIs) or selective inhibitors of norepinephrine reuptake (SNRI) as the first-line treatment, the actual effectiveness of pharmacological treatments barely exceeds 60\% effectiveness (Bayley et al., 2013). These data allows us to propose that cognitive behavioral therapy along with pharmacological treatment may have a greater impact in improving the symptoms of patients with PTSD.

\section{Acknowledgments}

This work was supported by the grant PAPIIT RN302512.

\section{References}

Arnsten, A.F. (2000). Through the looking glass: differential noradenergic modulation of prefrontal cortical function. Neural Plast, 7, 133-46.

Arnsten, A.F. (2009). Stress signalling pathways that impair prefrontal cortex structure and function. Nat Rev Neurosci, 10, 410-22.

Aston-Jones, G., Rajkowski, J., \& Cohen, J. (1999). Role of locus coeruleus in attention and behavioral flexibility. Biol Psychiatry, 46, 1309-20.

Bailey, C.R., Cordell, E., Sobin, S.M., \& Neumeister, A. (2013). Recent progress in understanding the pathophysiology of post-traumatic stress disorder: implications for targeted pharmacological treatment. CNS Drugs, 27, 221-32.

Birnbaum, S., Gobeske, K.T., Auerbach, J., Taylor, J.R., \& Arnsten, A.F. (1999). A role for norepinephrine in stress-induced cognitive deficits: alpha-1adrenoceptor mediation in the prefrontal cortex. Biol Psychiatry, 46, 1266-74.

Bremner, J.D. (2005). Effects of traumatic stress on brain structure and function: relevance to early responses to trauma. J Trauma Dissociation, 6, 51-68.

Bremner, J.D. (2006). Traumatic stress: effects on the brain. Dialogues Clin Neurosci, 8, 445-61. 
Carrasco, G. A., \& Van de Kar, L. D. (2003). Neuroendocrine pharmacology of stress. Eur J Pharmacol, 463(1-3), 235-272.

Gurvits, T. V., Shenton, M. E., Hokama, H., Ohta, H., Lasko, N. B., Gilbertson, M. W., . . Pitman, R. K. (1996). Magnetic resonance imaging study of hippocampal volume in chronic, combat-related posttraumatic stress disorder. Biol Psychiatry, 40(11), 1091-1099 doi: 10.1016/S0006-3223(96)00229-6

Holmes, A., \& Wellman, C.L. (2009). Stress-induced prefrontal reorganization and executive dysfunction in rodents. Neurosii Biobehav Rev, 33, 773-83.

Hubbard, D.T., Nakashima, B.R., Lee, I., \& Takahashi, L.K. (2007). Activation of basolateral amygdala corticotropin-releasing factor 1 receptors modulates the consolidation of contextual fear. Neuroscience, $150,818-28$.

Humphreys, K.L., Sauder, C.L., Martin, E.K., \& Marx, B.P. (2009). Tonic immobility in childhood sexual abuse survivors and its relationship to posttraumatic stress symptomatology. J Interpers Violence, 25, 358-73.

Keltner, N.L., \& Dowben, J.S. (2007). Psychobiological substrates of posttraumatic stress disorder--part I. Perspect Psychiatr Care, 43, 97-101.

Lanius, R.A., Vermetten, E., Loewenstein, R.J., Brand, B., Schmahl, C., Bremner, J.D., \& Spiegel, D. (2010). Emotion modulation in PTSD: Clinical and neurobiological evidence for a dissociative subtype. Am J Psychiatry, 167, 640-7.

Li, B.M., \& Mei, Z.T. (1994). Delayed-response deficit induced by local injection of the alpha 2 -adrenergic antagonist yohimbine into the dorsolateral prefrontal cortex in young adult monkeys. Behav Neural Biol, 62, 134-9.

Li, B.M., Mao, Z.M., Wang, M., \& Mei, Z.T. (1999). Alpha-2 adrenergic modulation of prefrontal cortical neuronal activity related to spatial working memory in monkeys. Neuropsychopharmacology, 21, 601-10.
Michelsen, K.A., van den Hove, D.L., Schmitz, C., Segers, O., Prickaerts, J., \& Steinbusch, H.W. (2007). Prenatal stress and subsequent exposure to chronic mild stress influence dendritic spine density and morphology in the rat medial prefrontal cortex. BMC Neurosci, 8, 107.

Mills, K. (2013). Treatment of comorbid substance dependence and posttraumatic stress disorder. $J A M A, 310,482-3$.

Peskind, E.R., Bonner, L.T., Hoff, D.J., \& Raskind, M.A. (2003). Prazosin reduces trauma-related nightmares in older men with chronic posttraumatic stress disorder. J Geriatr Psychiatry Neurol, 16, 165-71.

Petrov, T., Krukoff T.L., \& Jhamandas J.H. (1994). Chemically defined collateral projections from the pons to the central nucleus of the amygdala and hypothalamic paraventricular nucleus in the rat. Cell Tissue Res. 277(2), 289-95.

Pfaff, D. W., Silva, M. T., \& Weiss, J. M. (1971). Telemetered recording of hormone effects on hippocampal neurons. Science, 172(3981), 394-5.

Protopopescu, X., Pan, H., Tuescher, O., Cloitre, M., Goldstein, M., Engelien, W. ... Stern, E. (2005). Differential time courses and specificity of amygdala activity in posttraumatic stress disorder subjects and normal control subjects. Biol Psychiatry, 57(5), 464-73.

Radley, J. J., Rocher, A.B., Miller, M., Janssen, W.G., Liston, C., Hof, P.R., McEwen, B.S., \& Morrison, J.H. (2006). Repeated stress induces dendritic spine loss in the rat medial prefrontal cortex. Cereb Cortex, 16, 313-20.

Ramos, B. P., Colgan, L., Nou, E., Ovadia, S., Wilson, S. R., \& Arnsten, A. F. (2005). The beta-1 adrenergic antagonist, betaxolol, improves working memory performance in rats and monkeys. Biol Psychiatry, 58(11), 894-900. doi: 10.1016/j. biopsych.2005.05.022

Raskind, M.A., Thompson, C., Petrie, E.C., Dobie, D.J., Rein, R.J., Hoff, D.J., McFall, M.E. and Peskind, E.R. (2002). Prazosin reduces nightmares in combat veterans with 
posttraumatic stress disorder. J Clin Psychiatry, 63, 565-8.

Shestatzky, M., Greenberg, D., \& Lerer, B. (1998). A controlled trial of phenelzine in posttraumatic stress disorder. Psychiatry Res. 24(2), 149-55.

Shin L.M., Orr SP, Carson M.A., Rauch S.L., Macklin M.L., Lasko N.B., \& Pitman R.K. (2004). Regional cerebral blood flow in the amygdala and medial prefrontal cortex during traumatic imagery in male and female Vietnam veterans with PTSD. Arch Gen Psychiatry, 61(2), 168-76.

Shin, L.M., Wright, C.I., Cannistraro, P.A., Wedig, M.M., McMullin, K., Martis, B. ... Rauch, S.L. (2005). A functional magnetic resonance imaging study of amygdala and medial prefrontal cortex responses to overtly presented fearful faces in posttraumatic stress disorder. Arch Gen Psychiatry, 62(3), 273-81.

Squire, L.R., \& Zola-Morgan, S. (1991). The medial temporal lobe memory system. Science, 253(5026), 1380-6.

Stein, M. B., Koverola, C., Hanna, C., Torchia, M. G., \& McClarty, B. (1997). Hippocampal volume in women victimized by childhood sexual abuse. Psychol Med, 27(4), 951-959.

Uno H., Eisele S., Sakai A., Shelton S., Baker E., DeJesus O., \& Holden J. (2004). Neurotoxicity of glucocorticoids in the primate brain. Horm Behav, 28(4), 336-48.
Van de Kar, L. D., \& Blair, M. L. (1999). Forebrain pathways mediating stress-induced hormone secretion. Front Neuroendocrinol, 20(1), 1-48. doi: 10.1006/frne.1998.0172

Vermetten, E., Schmahl, C, Southwick, S.M., \& Bremner, J.D. (2007). Positron tomographic emission study of olfactory induced emotional recall in veterans with and without combat-related posttraumatic stress disorder. Psychopharmacol Bull, 40(1), 8-30.

Wang, M., Ramos, B.P., Paspalas, C.D., Shu, Y., Simen, A., Duque, A. ... Arnsten, A.F. (2007). Alpha2Aadrenoceptors strengthen working memory networks by inhibiting cAMP-HCN channel signaling in prefrontal cortex. Cell, 129, 397-410.

Womble, A.L. (2013). Effects of ketamine on major depressive disorder in a patient with posttraumatic stress disorder. $A A N A J, 81,118-9$.

Zhang, J., Tan, Q., Yin, H., Zhang, X., Huan, Y., Tang, L. \& Li, L. (2011). Decreased gray matter volume in the left hippocampus and bilateral calcarine cortex in coal mine flood disaster survivors with recent onset PTSD. Psychiatry Res, 192(2), 84-90. doi: 10.1016/j.pscychresns.2010.09.001

Received: March $4^{\text {th }}, 2014$

Accepted: August 20 $0^{\text {th }}, 2014$ 